(C) (C) 2016 IEEE. Personal use of this material is permitted. Permission from IEEE must be obtained for all other uses, in any current or future media, including reprinting/republishing this material for advertising or promotional purposes, creating new collective works, for resale or redistribution to servers or lists, or reuse of any copyrighted component of this work in other works. 


\title{
Control of a Master-Slave Pneumatic System for Teleoperated Needle Insertion in MRI
}

\author{
E. Franco*, M. Rea, W. M. Gedroyc, M. Ristic, Member, IEEE
}

\begin{abstract}
This paper presents the control of a pneumatically actuated master-slave system intended for teleoperated needle insertion in the liver under MRI-guidance. It addresses the challenge of achieving accurate needle positioning and force feedback to the operator in the case of pneumatic actuation with significant friction. Using time delay position control as the basis, we investigate force feedback via impedance control and admittance control. For impedance control, we propose a new adaptive friction compensation algorithm that only requires a single tuning parameter. Experiments on a 1 DOF prototype system using silicone rubber phantoms with distinct densities highlight the differences between impedance control and admittance control, and demonstrate superior performance compared to a traditional impedance control scheme.
\end{abstract}

Index Terms - Force control, Medical Robotics, Pneumatic Systems, Adaptive Control

\section{INTRODUCTION}

$\mathrm{T}$ ELEOPERATED needle insertion in MRI-guided percutaneous interventions offers higher targeting accuracy and substantial time saving compared to conventional manual procedures owing to simultaneous imaging and closed loop control. Additionally, differently from fully automated needle insertions, the clinicians remain in control. While this strategy is not yet viable for all MRIguided procedures and all needle types [1], recently proposed systems have attempted to provide this capability [2], [3]. Previous designs of master-slave robotic systems utilized ultrasonic and piezoelectric motors for the actuation of the needle insertion stage on the slave unit because of their compact size and high torques [4]-[7]. Yet, these actuators employ electric currents and metallic parts that can degrade the MR images [8] and may only qualify as MRI-conditional [9]. In order to reduce image degradation, specially designed motor drivers were employed [3], and the ultrasonic motors were kept outside the imaging volume [10]. Instead, pneumatic actuation can be designed to only employ nonmetallic materials and can qualify as MRI-safe [2]. Pneumatic actuation is clean, safe, and well suited for force control, as discussed in [1], [11] and references therein.

This work was supported by the i4i Grant II-AR-1109-11011.

E. Franco and M. Ristic are with the Mechanical Engineering Department, Imperial College London (e-mail: ef1311@imperial.ac.uk, m.ristic@imperial.ac.uk).

M. Rea and W. M. Gedroyc are with the Institute of Clinical Science, MRI Department, St Mary's Campus (e-mail: marc.rea@imperial.ac.uk, w.gedroyc@imperial.ac.uk).
However, commercially available pneumatic cylinders typically have high friction which makes position control and force control particularly challenging.

Differently from previous teleoperation systems for MRI guided intervention [4]-[7], [12], pneumatic actuation is employed here in both the master unit, which sits next to the operator, and in the slave unit, which is intended to operate in the MRI scanner [13]. This design choice allows using the system in the MRI room with minimal electromagnetic interference, but requires the accurate position and force control of the pneumatic cylinders. Our previous work [14] has shown that Time Delay Control (TDC) is well suited for the position control of pneumatic cylinders with significant friction. This work investigates how this controller can be used as the basis for teleoperated needle insertion, which involves providing a force feedback to the operator. Our main goal is the teleoperated needle insertion in roboticassisted interventions of liver tumors under MRI-guidance. In this application, the real time force feedback is intended to complement the intraoperative MR images, which are typically not available in real time. A detailed review of the needle insertion forces is reported in [15] and included references, to which we refer throughout the paper.

The first contribution of this work is the evaluation of two control strategies for this pneumatic system in order to provide force feedback: impedance control and admittance control. In teleoperation systems for the MRI environment, impedance control, which accepts a motion input and produces a force output, has been previously proposed for specially designed low-friction pneumatic cylinders [4]. Conversely, admittance control, which accepts a force input and produces a motion output, has been chosen for non-backdrivable actuators [6]. This work investigates the performance of both control strategies in the presence of significant friction in the pneumatic actuators. While admittance control appears promising because it is insensitive to the dynamics of the actuators, we postulate that the performance of impedance control could be enhanced with adaptive friction compensation. The position control of the slave unit, and in case of admittance control also of the master unit, is achieved with the TDC algorithm [14].

The second contribution of this work is a new adaptive algorithm employed to compensate the friction forces of the master piston as part of the impedance control scheme. The algorithm is an improved version of our controller [13] and it requires only one parameter. Experiments on silicone rubber 


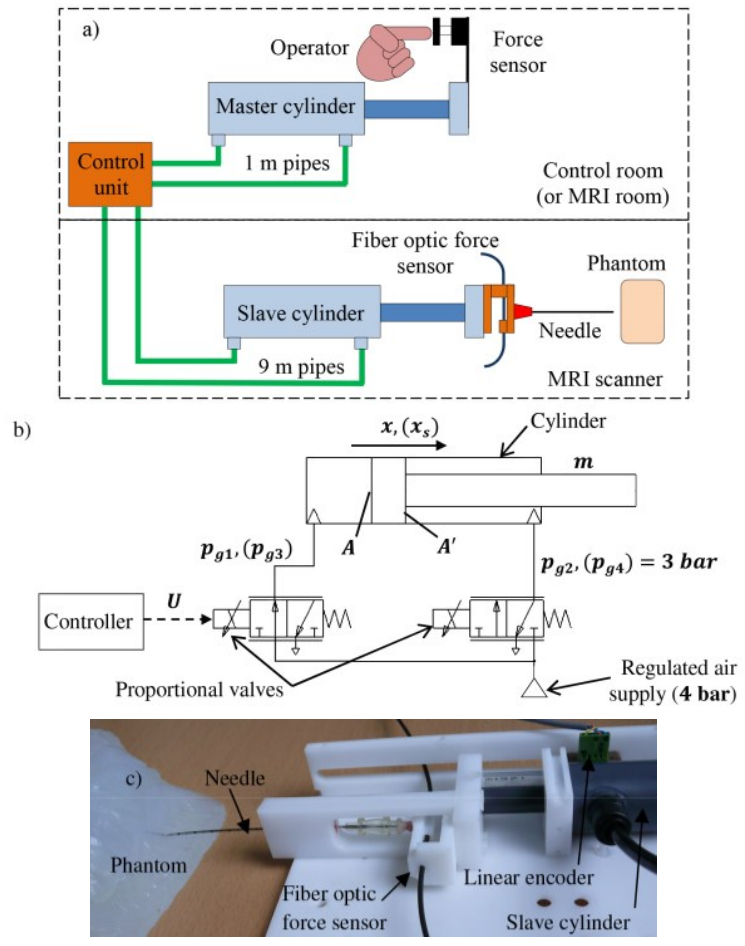

Fig. 1. Schematic of the master-slave system (a); pneumatic diagram of master (slave) cylinder (b); 1 DOF prototype of the slave unit (c).

phantoms highlight the differences between impedance control with friction compensation and admittance control, and demonstrate their benefits over a baseline controller.

\section{SYSTEM OVERVIEW}

This section gives an overview of the prototype, which consists of identical master and slave units, actuated by commercially available double-acting plastic pneumatic cylinders (AC-111707-501, IPS Inc.) powered using proportional valves (Tecno Basic, PS120000-080-010, Hoerbiger). Each proportional valve is equipped with a pressure sensor and controls the output pressure with an onboard PID scheme. The piston position is measured with optical encoders (EM1-250, US Digital), while the piston velocity is calculated by discrete differentiation and low pass filtering. The master is typically located in the control room and sits next to the control unit containing the proportional valves, while the slave is connected to the control unit using $9 \mathrm{~m}$ long supply lines. A $9 \mathrm{~m}$ long shielded cable and a waveguide adapter [1] connect the encoder on the slave to the control unit. If the specific application requires it, the master can also be operated in the scanner room.

The needle insertion force on the slave unit is measured with a specially designed fiber optic force sensor [13]. The sensor consists of a rapid-prototyped cantilever structure in which a segmented optic fiber acts as sensitive element. The needle insertion force produces a deflection of the cantilever structure and of the optic fiber. The variation in the measured light intensity is proportional to the applied force. The sensor has $0.1 \mathrm{~N}$ resolution, $15 \mathrm{~N}$ range, and $0.7 \mathrm{~N}$ accuracy. This performance was found to be satisfactory in experiments involving force feedback on silicone rubber phantoms. The sensor design and calibration are presented in detail in [13]. If the master is located in the control room, the operator force can be measured with a commercially available sensor (FSG15N1A, Honeywell, USA). A simplified schematic of the system, the detail for one cylinder, and the 1 DOF prototype of the slave unit are depicted in Fig. 1.

\section{CONTROLLer Design}

This section presents two control architectures for the master-slave system: impedance control and admittance control. Both approaches rely on the accurate position control of the slave piston, which is achieved here with an improved TDC scheme. TDC assumes bounded variation of the disturbances, which is valid if the sampling interval is small compared to the system dynamics. The disturbances are compensated using previous values of the control input and of the state variables. The TDC algorithm was presented in detail in [14] and will only be briefly summarized here:

$$
\begin{aligned}
& U=U^{\prime}+u_{2} \\
& U^{\prime}=\left(\alpha S+\ddot{x}_{D}+\lambda\left(\dot{x}_{D}-\dot{x}_{S}\right)-\ddot{x}_{S}(t-T)\right) m / A+ \\
& +U^{\prime}(t-T) \\
& u_{2}=\left\{\begin{array}{cl}
c(\operatorname{sg}(S)) & |S| \geq e \\
c(S / e) & |S|<e
\end{array}\right.
\end{aligned}
$$

The control input $U$ sets the pressure $p_{g 3}$ in the cylinder back chamber, while $p_{g 4}$ is set constant (Fig. 1b). The term $U^{\prime}$ 'represents the linear part of the control law. The switching term $u_{2}$ is introduced to enhance the position accuracy of the system in the presence of high Coulomb friction. The terms $x_{S}, \dot{x}_{S}, \ddot{x}_{S}, x_{D}, \dot{x}_{D}, \ddot{x}_{D}$ are the position, velocity, and acceleration of the slave piston and their prescribed values, while $\operatorname{sg}(\cdot)$ is the sign function. The terms $A, m$ are the effective piston area and the mass of piston and payload. The term $T$ is the sampling interval and the term $S$ is defined as:

$$
S=\lambda\left(x_{D}-x_{S}\right)+\dot{x}_{D}-\dot{x}_{s}
$$

The positive parameters $\alpha, \lambda, c, e$ are manually tuned as explained in [14]. The same values were employed for the master and the slave units in all tests (ref. Table II).

\section{A. Impedance Control}

Impedance control is the natural choice for pneumatic actuators, which are back-drivable and compliant [11]. Here this approach consists of the TDC position control on the slave unit and of a force control on the master. The operator manually defines the position of the master which becomes the setpoint for the slave. At the same time the needle insertion force measured on the slave is reflected on the master in real time (Fig. 2).

This section focuses on the force control algorithm employed on the master piston. The master is modelled as:

$\left\{\begin{array}{l}\dot{x_{1}}=x_{2} \\ \dot{x_{2}}=\frac{A p_{g 1}-A^{\prime} p_{g 2}+F_{u}-\gamma x_{2}-F_{a} \operatorname{sg}\left(x_{2}\right)-F_{t} \delta\left(x_{2}\right)}{m}\end{array}\right.$

The piston position $x$ and velocity $\dot{x}$ are indicated with $x_{1}$ 


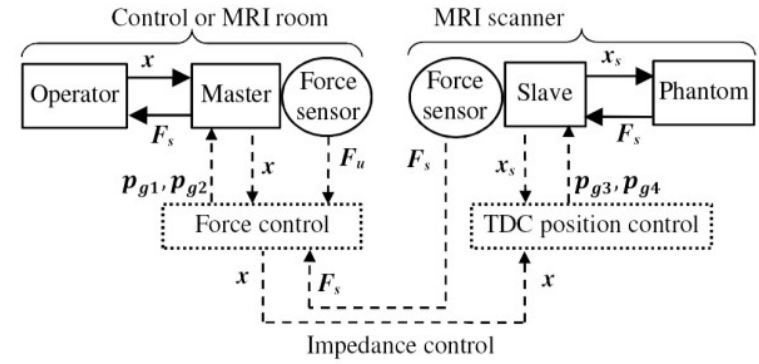

Fig. 2. Impedance control architecture of the master-slave system: solid lines represent physical interactions; dashed lines represent signals.

and $x_{2}$. The terms $A, A^{\prime}$ are the effective piston areas (ref. Fig. 1b). The terms $p_{g 1}, p_{g 2}$ are the pressures in the back and in the front cylinder chambers relative to atmosphere. The term $F_{u}$ is the operator force, while the terms $\gamma, F_{a}, F_{t}$ are the unknown coefficients of viscous friction, of Coulomb friction, and of stiction. We adopt a simplified friction model which employs the sign function $\operatorname{sg}(\cdot)$ for Coulomb friction and the delta function $\delta(\cdot)$ for stiction, that we define as:

$$
\operatorname{sg}\left(x_{2}\right)=\left\{\begin{array}{rl}
-1 & x_{2}<0 \\
0 & x_{2}=0 ; \\
1 & x_{2}>0
\end{array} \quad \delta\left(x_{2}\right)= \begin{cases}1 & x_{2}=0 \\
0 & x_{2} \neq 0\end{cases}\right.
$$

Since the movements of master and slave are relatively slow $(<10 \mathrm{~mm} / \mathrm{s})$ [13], the pressure dynamics of the supply pipes and the transient behavior of the proportional valves may be neglected [8], [16]. Consequently, $p_{g 1}, p_{g 2}$ are assumed equal to the output of the proportional valves at steady state.

The aim of the force controller is to reflect the insertion force $F_{S}$ on the master so that:

$$
F_{u}=F_{s}
$$

A control law which contains the unknown terms $\gamma, F_{a}, F_{t}$, can be obtained substituting (5) into (3) and solving for $p_{g 1}$ :

$$
p_{g 1}=\frac{A^{\prime} p_{g 2}-F_{s}+\gamma x_{2}+F_{a} \operatorname{sg}\left(x_{2}\right)+F_{t} \delta\left(x_{2}\right)+m \dot{x}_{2}}{A}
$$

In [4], a force controller for a specially designed low-friction pneumatic master was obtained assuming negligible inertial and friction forces. Keeping $p_{g 2}$ constant for simplicity, equation (6) becomes under this assumption:

$$
p_{g 1}=\left(A^{\prime} p_{g 2}-F_{s}\right) / A
$$

The force controller (7) on the master, together with the TDC position control (1) on the slave, represents the baseline impedance control scheme against which the controllers investigated in this work were evaluated (ref. Section IV).

While the inertial forces can be small for slow movements and for a small mass $m$, the friction forces in commercially available cylinders [1] can be comparable to the needle insertion forces [15] and represent the biggest challenge from a control perspective. Consequently, an adaptive algorithm is proposed to compensate Coulomb friction, viscous friction, and stiction. The controller is designed using the Immersion and Invariance method (I\&I) [17], which employs the sum of a state dependent function $\beta_{i}$ and of a state independent function $\hat{\eta}_{i}$ to estimate an unknown parameter $\eta_{i}$. This method was chosen because of its modularity and since it requires a limited number of parameters, which simplifies tuning [17]. In order to construct the I\&I adaptive control law, the three estimation errors $z_{1}, z_{2}, z_{3}$ are defined as:

$$
\left\{\begin{array}{c}
z_{1}=\left(\hat{\gamma}+\beta_{1}\right)-\gamma \\
z_{2}=\left(\hat{F}_{a}+\beta_{2}\right)-F_{a} \\
z_{3}=\left(\widehat{F}_{t}+\beta_{3}\right)-F_{t}
\end{array}\right.
$$

The three unknown parameters $\gamma, F_{a}, F_{t}$ are estimated by the functions $\left(\hat{\gamma}+\beta_{1}\right),\left(\hat{F}_{a}+\beta_{2}\right),\left(\hat{F}_{t}+\beta_{3}\right)$. These six terms are defined in order to ensure convergence of the estimation errors to zero, which is achieved using a Lyapunov approach. A positive definite candidate function $V$ is then chosen as:

$$
V=\left(z_{1}^{2}+z_{2}^{2}+z_{3}^{2}\right) / 2
$$

The estimation errors $z_{1}, z_{2}, z_{3}$ converge to zero if the adaptation law makes the derivative $\dot{V}$ negative definite:

$$
\dot{V}=z_{1} \dot{z_{1}}+z_{2} \dot{z_{2}}+z_{3} \dot{z_{3}}<0
$$

Differentiating (8) and substituting (3) we obtain for $\dot{z}_{1}$ :

$$
\begin{gathered}
\dot{z_{1}}=\dot{\hat{\gamma}}+\frac{\partial \beta_{1}}{\partial x_{1}} x_{2}+\frac{\partial \beta_{1}}{\partial x_{2}}\left(A p_{g 1}-A^{\prime} p_{g 2}+F_{u}\right) / m \\
-\frac{\partial \beta_{1}}{\partial x_{2}}\left(\left(\hat{\gamma}+\beta_{1}-z_{1}\right) x_{2}+\left(\hat{F}_{a}+\beta_{2}-z_{2}\right) \operatorname{sg}\left(x_{2}\right)\right) / m \\
-\frac{\partial \beta_{1}}{\partial x_{2}}\left(\left(\hat{F}_{t}+\beta_{3}-z_{3}\right) \delta\left(x_{2}\right)\right) / m
\end{gathered}
$$

Similar expressions are obtained for $\dot{z_{2}}, \dot{z_{3}}$, where $\beta_{2}, \beta_{3}$ replace $\beta_{1}$ and $\dot{\hat{F}_{a}}, \dot{\hat{F}}_{t}$ replace $\dot{\hat{\gamma}}$. We now choose the terms $\dot{\hat{\gamma}}, \dot{\hat{F}_{a}}, \dot{\hat{F}}_{t}$ so that the derivatives $\dot{z_{1}}, \dot{z_{2}}, \dot{z_{3}}$ (12) only contain the state dependent terms $\beta_{1}, \beta_{2}, \beta_{3}$ and the errors $z_{1}, z_{2}, z_{3}$ but are independent of $\hat{\gamma}, \hat{F}_{a}, \hat{F}_{t}$ themselves:

$$
\left\{\begin{array}{l}
\dot{z_{1}}=\frac{\partial \beta_{1}}{\partial x_{2}}\left(z_{1} x_{2}+z_{2} \operatorname{sg}\left(x_{2}\right)+z_{3} \delta\left(x_{2}\right)\right) / m \\
\dot{z_{2}}=\frac{\partial \beta_{2}}{\partial x_{2}}\left(z_{1} x_{2}+z_{2} \operatorname{sg}\left(x_{2}\right)+z_{3} \delta\left(x_{2}\right)\right) / m \\
\dot{z_{3}}=\frac{\partial \beta_{3}}{\partial x_{2}}\left(z_{1} x_{2}+z_{2} \operatorname{sg}\left(x_{2}\right)+z_{3} \delta\left(x_{2}\right)\right) / m
\end{array}\right.
$$

Exploiting the structure of (12), the partial derivatives of $\beta_{1}, \beta_{2}, \beta_{3}$ are chosen as:

$$
\begin{aligned}
& \frac{\partial \beta_{1}}{\partial x_{2}}=-\theta x_{2} m \\
& \frac{\partial \beta_{2}}{\partial x_{2}}=-\theta \operatorname{sg}\left(x_{2}\right) m \\
& \frac{\partial \beta_{3}}{\partial x_{2}}=-\theta \delta\left(x_{2}\right) m
\end{aligned}
$$

where $\theta>0$ is a single tuning parameter. Substituting (12) and (13) into (10) and regrouping the terms, we obtain:

$$
\dot{V}=-\theta\left(z_{1} x_{2}+z_{2} \operatorname{sg}\left(x_{2}\right)+z_{3} \delta\left(x_{2}\right)\right)^{2} \leq 0
$$

From (4), when $x_{2} \neq 0, \dot{V}=0$ only if $z_{1}, z_{2}=0$, while when 
$x_{2}=0, \dot{V}=0$ only if $z_{3}=0$. The convergence of (14) can be proved invoking LaSalle's invariance principle. The adaptation law for $\beta_{1}, \beta_{2}, \beta_{3}$ is obtained integrating (13):

$$
\begin{aligned}
& \beta_{1}=-\theta x_{2}^{2} m / 2 \\
& \beta_{2}=-\theta\left|x_{2}\right| m \\
& \beta_{3}=0
\end{aligned}
$$

The derivatives $\dot{\hat{\gamma}}, \dot{\hat{F}_{a}}, \dot{\hat{F}_{t}}$ are found substituting (12) and (13) into (11) and into its paired equations for $\dot{z_{2}}, \dot{z_{3}}$ :

$$
\begin{gathered}
\dot{\hat{\gamma}}=\theta x_{2}\left(A p_{g 1}-A^{\prime} p_{g 2}+F_{u}-\left(\hat{\gamma}+\beta_{1}\right) x_{2}\right) \\
-\theta x_{2}\left(\left(\hat{F}_{a}+\beta_{2}\right) \operatorname{sg}\left(x_{2}\right)+\left(\hat{F}_{t}+\beta_{3}\right) \delta\left(x_{2}\right)\right) \\
\hat{F}_{a}=\theta \operatorname{sg}\left(x_{2}\right)\left(A p_{g 1}-A^{\prime} p_{g 2}+F_{u}-\left(\hat{\gamma}+\beta_{1}\right) x_{2}\right) \\
-\theta \operatorname{sg}\left(x_{2}\right)\left(\left(\hat{F}_{a}+\beta_{2}\right) \operatorname{sg}\left(x_{2}\right)+\left(\hat{F}_{t}+\beta_{3}\right) \delta\left(x_{2}\right)\right) \\
\hat{F}_{t}=\theta \delta\left(x_{2}\right)\left(A p_{g 1}-A^{\prime} p_{g 2}+F_{u}-\left(\hat{\gamma}+\beta_{1}\right) x_{2}\right) \\
-\theta \delta\left(x_{2}\right)\left(\left(\hat{F}_{a}+\beta_{2}\right) \operatorname{sg}\left(x_{2}\right)+\left(\hat{F}_{t}+\beta_{3}\right) \delta\left(x_{2}\right)\right)
\end{gathered}
$$

Finally, the control law for the master cylinder under the assumption of negligible inertial forces is obtained from (6) where the friction forces are replaced by their estimates (8):

$$
\begin{gathered}
p_{g 1}=\left(A^{\prime} p_{g 2}-F_{s}+\left(\hat{\gamma}+\beta_{1}\right) x_{2}\right) / A \\
+\left(\left(\hat{F}_{a}+\beta_{2}\right) \operatorname{sg}\left(x_{2}\right)+\left(\hat{F}_{t}+\beta_{3}\right) \delta\left(x_{2}\right)\right) / A
\end{gathered}
$$

In particular, $\beta_{1}, \beta_{2}, \beta_{3}$ are defined in (15), while $\hat{\gamma}, \hat{F}_{a}, \hat{F}_{t}$ are computed integrating (16).

\section{B. Admittance Control}

With admittance control, the operator ideally does not experience the friction of the master cylinder, consequently this approach is promising for this system. In this case, the TDC position control (1) is employed both on the slave unit and on the master unit (Fig. 3). In particular, the reference position $x_{D}$ for the master is calculated based on the operator force $F_{u}$ and on the needle insertion force $F_{s}$ :

$$
F_{u}-F_{s}=m_{v} \ddot{x}_{D}+\gamma_{v} \dot{x}_{D}
$$

The terms $m_{v}, \gamma_{v}$ are the virtual mass and the virtual damping, which are empirically tuned in order to achieve a stable dynamics [18] (ref. Section IV). While small values of $m_{v}, \gamma_{v}$ can cause oscillations and instability, large values produce high inertial and damping forces on the operator, resulting in low transparency. Converting (18) into its discrete counterpart, the reference position $x_{D}$ can be computed for each sampling interval from the measured values of $F_{u}$ and $F_{s}$. Similarly to the impedance control, the master position $x_{1}$ is taken as the setpoint for the slave unit.

\section{EXPERIMENTAL RESULTS}

Similarly to [19], three silicone rubber phantoms (PlatSil GEL-10, Polytek) with different density (Table I) were used for the experiments together with a $14 \mathrm{G}$ coaxial needle (1.2 $\mathrm{mm}$ OD). Notably, higher insertion forces were recorded for

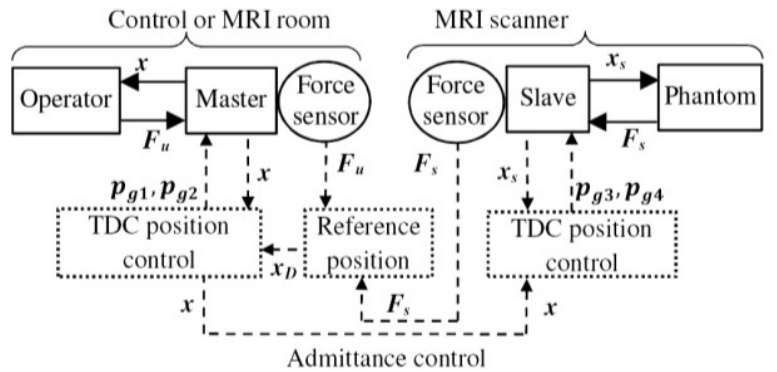

Fig. 3. Admittance control architecture of the master-slave system: solid lines represent physical interactions; dashed lines represent signals.

phantoms with higher density (ref. Table IV). Only positive insertion forces $F_{s}$ and operator forces $F_{u}$ were considered due to the current design of the optic fiber sensor [13]. The control algorithms (1), (7), (17), (18) were implemented on a microcontroller (mbed NHP LPC1768). A $1.5 \mathrm{kHz}$ sampling frequency was employed as in [14]. This value verifies the assumption of TDC (ref. Section III) without resulting in high control action that arises at higher frequencies due to quantization errors. All controller parameters are reported in Table II. The TDC parameters were manually tuned for high tracking accuracy and low overshoot as described in [14]. A moderate value of the parameter $\theta$ in (17) was chosen after some experimentation, since small values result in slow convergence of (14), while large values produce a jittery force feedback. While admittance control offers a simpler implementation compared to impedance control with friction compensation, it requires two tuning parameters instead of one. The parameters $m_{v}, \gamma_{v}$ were chosen empirically, starting with small values and increasing them gradually to achieve a smooth movement of the master [18]. Initial values of $m_{v}$ and $\gamma_{v}$ were the mass of the coaxial needle and the nominal viscous friction of the cylinders [1].

The pressure in both cylinders was limited between $0.3 \mathrm{bar}$ and 3 bar in order to provide the recommended 1 bar pressure drop across the proportional valves and since the nominal supply pressure in MRI suites is 4 bar. A dead band was employed in the impedance control so that the force feedback is activated only if $F_{u}>F_{M I N}$, where $F_{M I N}$ was set higher than the maximum errors of the force sensors. Similarly, in the admittance control the reference position $x_{D}$ is only updated if $F_{u}>F_{M I N}$ and $F_{u}>F_{s}$. This ensures that the master does not move against the operator due to

TABLE I. Phantom PRoperties

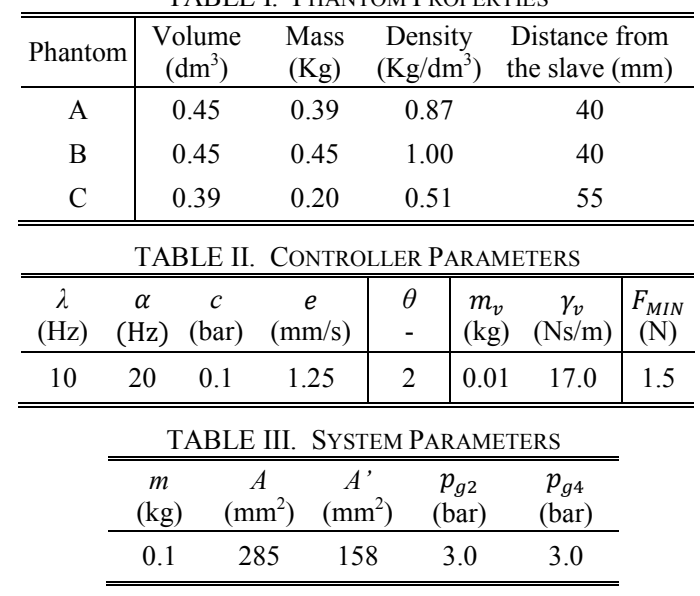



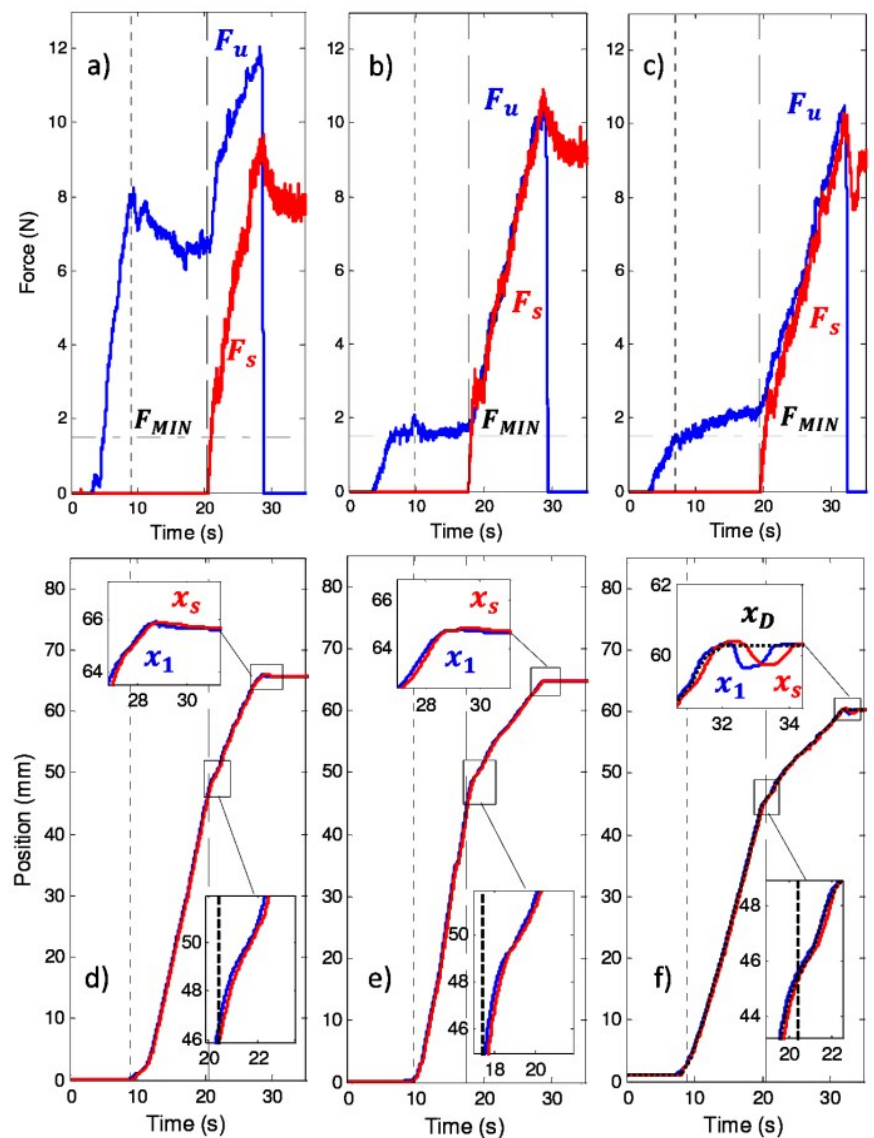

Fig. 4. Needle insertion on phantom A: forces (a) and positions (d) for the baseline controller (7); forces (b) and positions (e) for the adaptive controller (17); forces (c) and positions (f) for the admittance controller (18); Master position $x_{l}$ and operator force $F_{u}$ are plotted in blue; slave position $x_{s}$ and needle force $F_{s}$ are plotted in red. Reference position $x_{D}$ is the black dotted line in (f). The dead band $F_{M I N}$ is the grey horizontal centerline in (a), (b), (c).

measurement errors in the force sensors, which would result in incorrect haptic feedback.

The impedance control with adaptive friction compensation (17) and the admittance control (18) were compared with the baseline impedance control scheme (7) (ref. Section III). The position of the master and of the slave, the insertion force $F_{s}$, and the operator force $F_{u}$ for phantom A are depicted in Fig. 4. Similar results were obtained for the other phantoms, with larger forces recorded for phantom B and smaller forces for phantom $\mathrm{C}$ (Table IV). The dotted vertical line indicates the instant at which the master starts moving, while the dashed vertical line represents the initial contact of the needle with the phantom. The magnitude of the insertion force and of the insertion speed is representative of percutaneous clinical procedures [15]. Due to the significant friction of the commercially available cylinders used, the admittance control (18) was expected to result in a

TABLE IV. Force TRACKING ON MASTER CYLINDER

\begin{tabular}{|c|c|c|c|c|c|c|}
\hline \multirow[b]{3}{*}{ Phanto } & \multicolumn{4}{|c|}{ Impedance control } & \multirow{2}{*}{\multicolumn{2}{|c|}{$\begin{array}{l}\text { Admittance control } \\
\text { (18) }\end{array}$}} \\
\hline & \multicolumn{2}{|c|}{ Baseline (7) } & \multicolumn{2}{|c|}{ Adaptive (17) } & & \\
\hline & RMSE (N) & $\mathrm{F}_{\mathrm{s}}^{*}(\mathrm{~N})$ & $\operatorname{RMSE}(\mathrm{N})$ & $F_{\mathrm{s}}^{*}(\mathrm{~N})$ & RMSE (N) & $\mathrm{F}_{\mathrm{s}}^{*}(\mathrm{~N})$ \\
\hline A & 4.06 & 9.7 & 0.89 & 10.9 & 1.06 & 10.3 \\
\hline B & 3.61 & 14.2 & 1.07 & 14.7 & 1.31 & 12.7 \\
\hline $\mathrm{C}$ & 3.74 & 5.9 & 0.99 & 4.55 & 1.31 & 5.9 \\
\hline
\end{tabular}

Results refer to the interval where $F_{u}>0 . F_{\mathrm{s}}^{*}$ is the maximum needle force. lower force $F_{u}$ than the baseline impedance control (7). The results confirm this hypothesis and also show that the impedance control with adaptive friction compensation (17) is most effective in reducing the operator force. A lower force $F_{u}$ enhances the sensitivity of the operator to force variations [20] and may increase the ability to detect the tissues. In this case, the operator reacts reducing the insertion speed when the needle punctures the phantom, as shown in the magnified position plots at the bottom of Fig. 4. Due to the dead band, a force $F_{u}>F_{M I N}$ is still required to move the master. Above this value, which depends on the accuracy of the force sensors, the operator force $F_{u}$ is very close to the insertion force $F_{s}$. Notably, the insertion force does not fall to zero as the operator releases the master. This is expected since the slave maintains its position against the reaction forces of the phantom. The root-mean-square-error (RMSE) between $F_{u}$ and $F_{s}$ for $F_{u}>0$ is reported in Table IV. The RMSE for control schemes (17) and (18) is about one third of that calculated for the baseline impedance control (7). In particular, the smallest RMSE corresponds to the impedance control with adaptive friction compensation.

The magnified position plot at the top of Fig. $4 \mathrm{f}$ shows a first difference between impedance control and admittance control: with the admittance control the master springs back when $F_{u}$ instantly drops to zero. This behavior is due to the compliance of the pneumatic cylinders, accentuated by the low supply pressure. Consequently the slave, which follows the master position $x_{1}$, quickly retracts before settling to its final position, producing an oscillation in $x_{s}$ and in $F_{s}$. In general with this type of pneumatic systems, a large and rapid variation of $F_{u}$ can affect the master position $x_{1}$, which could differ substantially from its reference $x_{D}$. While the amplitude of the oscillation is minimal with the tuning adopted, this effect reduces transparency. A slight movement of the master when $F_{u}$ drops to zero is also noticeable with impedance control, although no oscillations are observed.

The second subtle difference between impedance control with adaptive friction compensation and admittance control is noticeable from the force plots: while in the former case the operator force before the insertion is almost constant (Fig. 4b), the admittance control shows a moderate but clear increase of $F_{u}$ during motion (Fig. 4c). Since before the contact with the phantom (vertical dashed line) the needle force $F_{s}$ is close to zero, $F_{u}$ balances the inertial and damping forces due to $m_{v}$ and $\gamma_{v}$ according to (18). Therefore, as showed in Table IV, the RMSE with admittance control (18) is slightly larger than with the impedance control (17), which
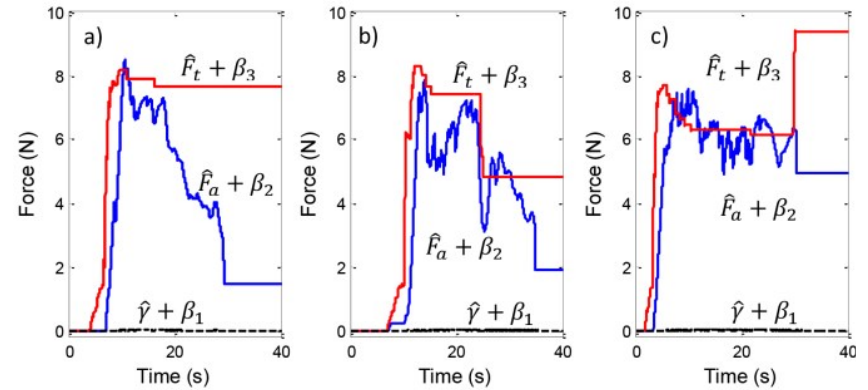

Fig. 5. Estimates of the friction coefficients: viscous friction $\left(\hat{\gamma}+\beta_{1}\right)$ is plotted in dashed black; Coulomb friction $\left(\hat{F}_{a}+\beta_{2}\right)$ is in solid blue; stiction $\left(\hat{F}_{t}+\beta_{3}\right)$ is in red. (a) Phantom A; (b) Phantom B; (c) Phantom C. 
indicates a lower transparency. While lower values of $m_{v}$ and $\gamma_{v}$ would reduce this effect, setting them to zero would compromise stability. In practice, the effects of $m_{v}$ and $\gamma_{v}$ with the tuning employed are not noticeable by the operator.

The estimated friction coefficients $\left(\hat{\gamma}+\beta_{1}\right),\left(\hat{F}_{a}+\right.$ $\left.\beta_{2}\right),\left(\hat{F}_{t}+\beta_{3}\right)$ are depicted in Fig. 5 for all phantoms. While the viscous friction coefficient is very low in the range of speed of interest, both stiction and Coulomb friction coefficients are in fact comparable to the needle insertion force, as postulated in Section III (ref. Table IV). As expected, Fig. 5 shows that stiction is slightly larger than Coulomb friction. In particular, Coulomb friction shows a high variability. This is due to the inertial forces of the master, that were neglected in the adaptation law (ref. Section III), and to the measurement noise in both force sensors. Furthermore, the calculation of the piston velocity through discrete differentiation is affected by quantization errors that are particularly noticeable at low speed.

Finally, the TDC position control (1) proved effective for this system (tracking error on the slave unit $\leq 1.3 \mathrm{~mm}$, RMSE $\leq 0.4 \mathrm{~mm}$ ) and appropriate for this application.

\section{CONCLUSION}

This paper presented the control of a pneumatic masterslave system intended for teleoperated needle insertion in MRI. Impedance control and admittance control were evaluated for this system, characterized by significant actuator friction. A TDC algorithm was employed for the position control of the pneumatic cylinders. For the impedance control, a new adaptive algorithm that requires a single tuning parameter was designed to compensate the friction forces of the master. The experiments showed that the impedance control with adaptive friction compensation and the admittance control are effective in the presence of significant friction. While both approaches proved superior compared to a baseline impedance control scheme, the lowest operator force and the smallest RMSE were recorded for impedance control with adaptive friction compensation. With the admittance control, the compliance of the pneumatic actuators was responsible for unwanted oscillations of the master under rapidly varying operator force. Additionally, the operator force showed a small but clear increase with the insertion speed during the free movement of the slave. Finally, the admittance control scheme employs two parameters and is therefore more difficult to tune. Overall, the impedance control with adaptive friction compensation proved the most suitable approach for our system.

Future work will explore more complex friction models and will include tests in the MRI scanner and using different phantoms. Of particular interest would be the ability to distinguish different types of tissues during needle insertion based on force feedback. Ultimately, we intend to integrate the teleoperated needle insertion in our pneumatically actuated robot for MRI-guided ablation of liver tumors.

\section{REFERENCES}

[1] E. Franco, D. Brujic, M. Rea, W. M. Gedroyc, and M. Ristic, "NeedleGuiding Robot for Laser Ablation of Liver Tumors Under MRI
Guidance," IEEE/ASME Trans. Mechatronics, vol. 21, no. 2, pp. 931944, Apr. 2016

[2] D. Stoianovici, C. Kim, G. Srimathveeravalli, P. Sebrecht, D. Petrisor, J. Coleman, S. B. Solomon, and H. Hricak, "MRI-safe robot for endorectal prostate biopsy," IEEE/ASME Trans. Mechatronics, vol. 19, no. 4, pp. 1289-1299, 2014.

[3] H. Su, W. Shang, G. Cole, G. Li, K. Harrington, A. Camilo, J. Tokuda, C. M. Tempany, N. Hata, and G. S. Fischer, "Piezoelectrically Actuated Robotic System for MRI-Guided Prostate Percutaneous Therapy," IEEE ASME Trans. Mechatron., vol. 20, no. 4, pp. 19201932, Aug. 2015.

[4] W. Shang, H. Su, G. Li, and G. S. Fischer, "Teleoperation system with hybrid pneumatic-piezoelectric actuation for MRI-guided needle insertion with haptic feedback," in IEEE International Conference on Intelligent Robots and Systems, 2013, pp. 4092-4098.

[5] R. Seifabadi, S. E. Song, A. Krieger, N. B. Cho, J. Tokuda, G. Fichtinger, and I. Iordachita, "Robotic system for MRI-guided prostate biopsy: Feasibility of teleoperated needle insertion and ex vivo phantom study," Int. J. Comput. Assist. Radiol. Surg., vol. 7, no. 2, pp. 181-190, 2012.

[6] Z. T. H. Tse, H. Elhawary, M. Rea, B. Davies, I. Young, and M. Lamperth, "Haptic needle unit for MR-guided biopsy and its control," IEEE/ASME Trans. Mechatronics, vol. 17, no. 1, pp. 183-187, 2012.

[7] B. Yang, S. Roys, U.-X. Tan, M. Philip, H. Richard, R. Gullapalli, and J. P. Desai, "Design, Development, and Evaluation of a Master-Slave Surgical System for Breast Biopsy under Continuous MRI," Int. J. Rob. Res., vol. 33, no. 4, pp. 616-630, Apr. 2014.

[8] B. Yang, U. X. Tan, A. McMillan, R. Gullapalli, and J. P. Desai, "Design and Control of a 1-DOF MRI Compatible Pneumatically Actuated Robot with Long Transmission Lines," IEEE ASME Trans Mechatron, vol. 16, no. 6, pp. 1040-1048, 2011.

[9] ASTM F2503-13 Standard Practice for Marking Medical Devices and Other Items for Safety in the Magnetic Resonance Environment. ASTM International, 2013.

[10] F. Sergi, A. C. Erwin, and M. K. OrMalley, "Interaction Control Capabilities of an MR-Compatible Compliant Actuator for Wrist Sensorimotor Protocols During fMRI," IEEE/ASME Trans. Mechatronics, vol. PP, no. 99, pp. 1-13, 2015.

[11] Y. Ningbo, C. Hollnagel, A. Blickenstorfer, S. S. Kollias, and R. Riener, "Comparison of MRI-Compatible Mechatronic Systems With Hydrodynamic and Pneumatic Actuation," Mechatronics, IEEE/ASME Trans., vol. 13, no. 3, pp. 268-277, 2008.

[12] R. Kokes, K. Lister, R. Gullapalli, B. Zhang, A. MacMillan, H. Richard, and J. P. Desai, "Towards a teleoperated needle driver robot with haptic feedback for RFA of breast tumors under continuous MRI," Med Image Anal, vol. 13, no. 3, pp. 445-455, Jun. 2009.

[13] E. Franco and M. Ristic, "Adaptive control of a master-slave system for teleoperated needle insertion under MRI-guidance," in 2015 23rd Mediterranean Conference on Control and Automation (MED), 2015, pp. 61-67.

[14] E. Franco and M. Ristic, "Time delay controller for the position control of a MRI-compatible pneumatic actuation with long supply lines," in 2014 IEEE/ASME International Conference on Advanced Intelligent Mechatronics, 2014, pp. 683-689.

[15] D. J. van Gerwen, J. Dankelman, and J. J. van den Dobbelsteen, "Needle-tissue interaction forces--a survey of experimental data.," Med. Eng. Phys., vol. 34, no. 6, pp. 665-80, 2012.

[16] B. Iranpanah, M. Chen, A. Patriciu, and S. Sirouspour, "A Pneumatically Actuated Target Stabilization Device for MRI-Guided Breast Biopsy," IEEE/ASME Trans. Mechatronics, vol. 20, no. 3, pp. 1288-1300, Jun. 2015.

[17] A. Astolfi and R. Ortega, "Immersion and invariance: A new tool for stabilization and adaptive control of nonlinear systems," IEEE Trans. Automat. Contr., vol. 48, no. 4, pp. 590-606, 2003.

[18] A. Achhammer, C. Weber, A. Peer, and M. Buss, "Improvement of model-mediated teleoperation using a new hybrid environment estimation technique," in 2010 IEEE International Conference on Robotics and Automation, 2010, pp. 5358-5363.

[19] A. M. Okamura, C. Simone, and M. D. O'Leary, "Force modeling for needle insertion into soft tissue.," IEEE Trans. Biomed. Eng., vol. 51, no. 10, pp. 1707-16, Oct. 2004.

[20] H. Tan, M. A. Srinivasan, B. Eberman, and B. Cheng, "Human Factors For The Design Of Force-Reflecting Haptic Interfaces," Dyn. Syst. Control, vol. 55, no. 1, pp. 353-359, 1994. 